\title{
Crisis sanitaria y crisis del trabajo: ¿una oportunidad en el caos?
}

Fabien Coutarel ${ }^{1}$; Valérie Pueyo 2 ; Marianne Lacomblez ${ }^{3}$; Catherine Delgoulet ${ }^{4}$; Béatrice Barthe ${ }^{5}$; Véronique Poète ${ }^{6}$; Alain Garrigou ; Bernard Dugué ${ }^{8}$; Christian Blatter ${ }^{9}$; Anne Grunstein ${ }^{10}$; Eric Liehrmann ${ }^{11}$; Bernard Michez ${ }^{12}$; Philippe Negroni ${ }^{13}$; Camille Thomas ${ }^{14}$.

\section{FALTAN FECHAS}

Resumen. En un contexto excepcional de confinamiento que ha llevado a un cambio en la vida personal y profesional de todos, la SELF inició la redacción de un texto, cuyo objetivo es contribuir a reflexiones y debates dentro de la comunidad de ergónomos y ergónomas, y en general de la sociedad.

Contrariamente a ciertas ideas preconcebidas, la ergonomía no consiste principalmente en el diseño de sillas, escritorios o cepillos de dientes "ergonómicos". El trabajo es un objeto central de la ergonomía, y los recursos técnicos asociados con él cubren solo algunas de las preguntas que éste plantea. El proyecto ergonómico consiste en percibir este Trabajo más allá de los aspectos técnicos: los valores, habilidades, relaciones, organizaciones, etc. que son sus componentes fundamentales, como lo es su inclusión en la sociedad y, por lo tanto, en la vida de todos y cada uno de nosotros.

Palabras clave: crisis sanitaria; crisis del trabajo; ergonomía.

\section{[en] Health crisis. Work crisis. An opportunity amidst the chaos?}

Abstract. The present context of health crisis and unprecedented lockdown is an opportunity for thinking, positiontaking, indignation and controversy. As a social activity, work redefines itself every day, according to circumstances. Work specialists, ergonomists included, observe and reflect.

Contrary to certain preconceived ideas, Ergonomics is not primarily about so-called "ergonomic" chairs, desks or toothbrushes. Work is a central object of ergonomics, and the technical resources associated with it cover only some of the questions it raises. The ergonomic project consists of perceiving this Work; and above and beyond the technical resources, it is the values, skills, relationships, organizations, etc. that are its pivotal components, as is its inclusion in society, and therefore in the lives of each and every one of us.

Keywords: health crisis, lockdown, ergonomy.

Sumario: Introducción. La ergonomía y el trabajo humano: hacer sociedad. La crisis sanitaria revela a todos los callejones sin salida y los ángulos muertos del trabajo de antes de la crisis. Retomar el control del Trabajo es posible. Cualquier ciudadano es responsable de concebir esta pandemia, como una avería o como una crisis, para y más allá del Trabajo. Bibliografía.

Maître de Conférences, Ergónomo, Universidad Clermont Auvergne, Laboratoire ACTé, Labex IMobS3 I SITE CAP 20-25, Administrador de la SELF, Vicepresidente del CE2.

Maîtresse de Conférences, Ergónomo, Universidad Lyon 2, Laboratoire Environnement Ville et Société, UMR 5600, Labex Intelligence des Mondes Urbains, Vice presidente de la SELF [Sociedad de Ergonomía de Lengua Francesa]

Profesora Emérita, Psicóloga del trabajo, Universidad de Porto, Miembro de la SELF

Profesora del CNAM, Titulaire de la Chaire d'Ergonomie, Conservatoire National des Arts et Métiers, Laboratoire CRTD, Directora del Gis-CREAPT, Miembro de la SELF

Maîtresse de Conférences, Ergónoma, Universidad Toulouse Jean Jaurès, Presidenta de la SELF

Consultora de Alternatives Ergonomiques, Ergónoma, Miembro de la SELF.

Profesor de Universidad, Bordeaux Population Health Center - Inserm U1219, Ergónomo, Universidad de Bordeaux, Président du CE2

Profesor e investigador, Ergónomo, Insituto Politécnico de Bordeaux, Presidente del CREE

Ergónomo retirado, antiguo jefe de la unidad de ergonomía y FOH de la SNCF, Tesorero de la SELF

Ergónoma, Miembro de la SELF

1 Ergónomo, Responsable el "pôle Approche Globale des Situations de Travail”, Instituto Nacional de Recherche en Santé et Sécurité au Travail, Vicepresidente de la SELF

12 Consultor de Ergotec, Ergónomo, Miembro de la SELF, Administrador Cinov Ergonomie, Presidente de la FEES

13 Consultor Sud Concept, Ergónomo, Secretario General de la SELF

14 Consultor e investigador Atitlan, Ergónomo, Miembro de SELF

Sociol. trab. n॰96 (2020): 17-21 
Cómo citar: Coutarel, F. et alii. Crisis sanitaria y crisis del trabajo: ¿una oportunidad en el caos? Sociología del Trabajo, $\mathrm{n}^{\circ} 96$ (2020), 17-21.

\section{Introducción*}

El contexto actual de crisis sanitaria y de confinamiento inédito de la población es la ocasión de reflexiones, de tomas de posiciones, de indignaciones, de controversias. El trabajo, en tanto que actividad social, se redefine él mismo todos los días, según las circunstancias. Los especialistas del trabajo, entre ellos los ergónomos, observan y se interrogan.

Contrariamente a ciertas ideas hechas, la ergonomía no es, en primer lugar, una historia de sillas, de oficinas o de cepillos de dientes, llamados 'ergonómicos'. El Trabajo es un objeto central de la ergonomía, y los medios técnicos que se le asocian no cubren más que una parte de las cuestiones que convoca. El proyecto de la ergonomía es el de concebir ese trabajo, y, más allá de los medios técnicos, son los valores, las competencias, las relaciones, las organizaciones, etc., los componentes decisivos, al igual que su inscripción en la sociedad, y por tanto, en la vida de cada uno y cada una.

\section{La ergonomía y el trabajo humano: hacer sociedad.}

Los ergónomos se inscriben en una tradición humanista que busca inicialmente adaptar el trabajo a los hombres y mujeres en toda du diversidad ${ }^{15}$, y esto, al correr del tiempo, con el fin de que no pierdan ni desgasten su vida mientras se la ganan, sino más bien al contrario: con el fin de que se desarrollen y construyan su salud, en y por el trabajo. En esta tradición, la idea principal es la de hacer las cosas de tal modo que el Trabajo sea humano, es decir que pueda garantizar y alimentar las necesidades vitales. Y si el nivel de remuneración y su constancia deben permitir el asegurar las necesidades elementales, nuestras necesidades vitales de humanos cubren también:

- la creación de un vínculo social y cultural, que permita hacer, tejer, enlazar, aprender, compartir, crear e innovar, con y para otros;

- la inscripción en una comunidad y un territorio, que te reconoce, te acepta, te protege y tiene necesidad de ti;

- el sentimiento de ser útil: producir bienes y servicios que tienen sentido, que valen a los ojos de los que cuentan.

Hay ahí, ciertamente palancas de potenciales logros bajamente utilizados en muchas organizaciones. Para decir las cosas de otra manera: se trata de un trabajo que ofrece a cada uno y cada una la posibilidad de vivirse como auténtico protagonista de la sociedad, y actor de unos logros que tienen sentido. Por ello es imperativo cuidar de ese trabajo y, a veces, mejorarlo; pensarlo, y a veces curarlo. La Ergonomía contribuye así, con otras, a dibujar los contornos de lo que podría ser una sociedad que permite verdaderamente eso, en escalas diferentes: hacer sociedad en el espacio de trabajo es hacer sociedad en el territorio y más allá. Relación de la ergonomía con la sociedad, vía el trabajo, es una preocupación original ${ }^{16}$, que esta crisis sanitaria reaviva.

\section{La crisis sanitaria revela a todos los callejones sin salida y los ángulos muertos del trabajo de antes de la crisis.}

El momento histórico que vivimos es un revelador extremadamente potente: esta pandemia procede de lo que Mauss, antropólogo, llama un "hecho social total", un fenómeno que "pone en cuestión la totalidad de la sociedad y de sus instituciones"17, que compromete a toda una sociedad, a todos sus miembros. Su comprensión supone no descomponer o diseccionar el fenómeno según las diversas dimensiones (biológica, histórica, política, jurídica, geográfica, demográfica, psicológica, económica...), puesto que es "considerando el todo conjunto, y conjuntamente, como [podremos] percibir lo esencial". La pandemia impone a todos la visión global y sistémica, reivindicada por la ergonomía tal y como nosotros la concebimos.

Y es gracias al trabajo de los trabajadores y trabajadoras de la salud, de la limpieza, de la educación, de la agricultura, de la alimentación, de la energía, de los transportes, de la información y de tantos otros confinados, trabajando a distancia, como nuestra sociedad "vigilante", afronta la pandemia. Muchos oficios que tienen que ver hoy con nues-

* Texto aparecido por primera vez en francés e inglés en la Web de la Sociedad de Ergonomía de Lengua Francesa [https://ergonomie-self.org/] el 26-05-2020, cedido por los autores a Sociología del Trabajo para su publicación una vez traducido al castellano y adaptado al formato de revista científica por Juan José Castillo.

15 Teiger, C. (1993) : 'L'approche ergonomique: du Travail humain à l'activité des hommes et des femmes au travail, Éducation Permanente, 116, pp.71-96.

16 Daniellou, F. : (2006): «Je me demanderais ce que la société attends de nous...», A propos des positions épistemologiques d'Alain Wisner, Travailler, $15,23-28$

17 Mauss, M. (1973): «Essai sur le don: forme et raison de l'échange dans les sociétés archaï ques», en Sociologie et Anthropologie, PUF, Collection Sociologie d'aujourd'hui, 143-279, pp. 274-275 
tra subsistencia estaban minusvalorados ${ }^{18}$ hasta ahora. Muchos y muchas de quienes aseguran nuestras necesidades alimentarias de confinados, aceptando riesgos, son, ampliamente, esos de los que la sociedad ha vaciado progresivamente su trabajo de las necesidades vitales de un trabajo humano, en el sentido desarrollado más arriba.

¡Qué paradoja! Pero, ¡qué fuente de esperanza, también, este confinamiento! Frente al caos, los basureros, los agentes de la limpieza y cajeras son hoy aplaudidas, reconocidas, reconocidos, factores de vínculos sociales; frente a los destrozos hospitalarios del virus, los cuidadores ven socialmente volver a ser socialmente aceptables sus alertas hace ya mucho tiempo inaudibles; frene a la mortalidad de los de más edad, los oficios de ayuda a las personas encarnan el único refugio de humanidad para acompañar el final de una vida.

Estos oficios reconquistan, en el tiempo de la crisis sanitaria, su Trabajo. Pero el precio, ¿es razonable? ¿En qué condiciones y con qué riesgos, con qué libertad de elección? ¿Les dejamos verdaderamente la opción? ¿Podemos aguantar que esta reconquista no dure más que el tiempo del paréntesis sanitario, por largo que sea?

Las opciones económicas que han sobredeterminado estos últimos años las evoluciones del trabajo y la sociedad no son extrañas a los efectos que constatamos hoy en día. Pensemos aquí en la mundialización, con su lote de subcontrataciones de bajo coste, de deslocalizaciones de la producción y los servicios o del cuasi monopolio de las multinacionales; pensemos también en la globalización de las cadenas de valor, y en la hiperespecialización de cada uno de los actores de la cadena. La opción del coste menor a corto plazo para el cliente se ha privilegiado, en detrimento de la calidad del trabajo: la hiperespecialización conduce a la desvalorización de los saberes en el trabajo, a la individualización de las tareas, al desmigajamiento de la actividad ${ }^{19}$, a la pérdida de sentido, a la calidad dificultada ${ }^{20}$. En detrimento de las sociedades mismas, es decir en detrimento del interés de la mayoría: más consumo, más transportes y polución; gran dependencia con contextos mundiales muy lejanos y poco influenciables. Cuando eso toca a las máscaras y los respiradores en tiempos de Covid19, la crisis del trabajo se convierte en un problema de subsistencia. Los costes del relanzamiento y las deudas contraídas para sobrepasarlo serán inmensos. Incluso económicamente para el cliente mismo, la elección del coste menor a corto plazo se hace discutible frente a las consecuencias de la pandemia.

Interroguemos la intensificación cada vez más grande del trabajo, combinando por ejemplo el mantenimiento de plazos cada vez más ajustados con una estandarización de los modos operatorios y un control incrementado de las operaciones realizadas: privando el trabajo de su complejidad, hasta el punto de que el término "mano de obra" no conserva hoy más que el sentido de un coste a reducir.

Miremos la omnipresencia de los imperativos gestionarios que implican sobre todo una programación 'ajustada' de los personales y la desaparición de los stocks. Asociados lo más a menudo con la ilusión de un trabajo nominal y controlado, estos imperativos conducen a los trabajadores a operar en condiciones siempre degradadas, por el hecho de las variabilidades inherentes al trabajo humano, que, porque son negadas, no son tomadas en cuenta seriamente. La ausencia de stocks, antes, durante y hacia debajo de las herramientas de producción, limitan los riesgos de una producción realizada y no vendida. Pero eso hace también el trabajo humano dependiente del mercado, dependencia que la flexibilización de los contratos y el tiempo de trabajo permite integrar: horarios desplazados, sumisión a tiempo parcial, contratos de corta duración, etc.

La revolución digital de nuestra sociedad sostiene ampliamente la banalización creciente de estos tiempos de trabajo atípicos, de la precarización social, incluida la familiar, ya que los equilibrios entre vida de familia y vida profesional son a veces sacudidos. Desde este punto de vista, el teletrabajo impuesto, simultáneamente con el cuidado de los niños, a los cuales esos mismos padres y madres deben, finalmente, asegurar una continuidad pedagógica, ofrece una experiencia masiva de las dificultades asociadas a las desregulaciones del sistema de actividades ${ }^{21}$.

Opciones todas que deshumanizan el Trabajo, tratado en ese caso como un coste y no como una baza del desarrollo individual, colectivo, local y del entorno. El "factor humano" es, como mucho, en el mejor caso, una variable de ajuste; y en el peor un factor de economías a hacer, en parte justificadas por las formas de descompromiso, que engendra. Los efectos nefastos de estas condiciones de trabajo sobre las personas son bien identificados en la literatura científica, y para algunos de ellos desde hace mucho tiempo. Pero son también ampliamente banalizados por los dispositivos de reparación que vienen a compensar las dificultades de salud confesadas, a veces duraderas, incluso con expectativas de vida limitadas.

Por supuesto, todo esto no concierne a todo el mundo. Pero las tendencias siguen siendo mayores y ampliamente dominantes. La explicitación, la intervención, la teorización, sobre todo en ergonomía, han permitido poner a la vista, las dinámicas subyacentes a estas concepciones de la 'excelencia' económica, con la vocación de sobrepasarlas, y superarlas, y no mantenerse en constataciones alarmistas, por pertinentes que sean. De todas maneras, los logros y éxitos locales han sido muy discretos a escala social, como para contribuir a una transformación radical del estado de las cosas.

\footnotetext{
8 Messing, K., Haentjens, C. y Doniol-Shaw, G.: "L'invisible nécessaire: l'activité de nettoyage des toilettes sur les trains de de voyageurs en gare", Le Travail Humain, 55, 4, 1992, pp. 353-370.

Friedmann G. (1956), Le Travail en miettes, Gallimard, (réed. 1964).

Clot, Y : (2010), Le Travail à cour, Paris, La Découverte.

Curie, J. , Hajjar, V., Baubios-Broye, A. (1990): «Psychopathologie du Travail ou dérégulation du système des activités» Perspectives psychiatriques, 22, pp.85-91.
} 


\section{Retomar el control del Trabajo es posible.}

Todo no es, sin embargo, tan sombrío, puesto que, en la experiencia de la crisis, un buen número de trabajadores han hecho la experiencia de situaciones de trabajo nuevas, solos o en colectivos existentes o (re)compuestos, desvelando a menudo otras maneras de hacer y de pensar el Trabajo y la Sociedad: solidarios, portadores del orgullo de ser útiles, auténticos, animados por valores morales y concepciones alternativas del "vivir juntos". Muy pequeñas empresas del textil modifican su producción para fabricar mascarillas; un sitio de una multinacional de la cosmética se lanza a la producción de un gel hidroalcohólico; enfermeras que adaptan trajes de pintor para fabricar blusas; fabricantes de automóviles que hacen respiradores; la panadería artesanal recluta para entregar a domicilio a las personas aisladas, etc. Esta reconfiguración de las maneras de hacer modifica todos los días lo cotidiano de los hospitales, de los EHPAD, del sector de la agricultura de verduras, del sector de edificios y obras públicas, y muchos otros. Es el trabajo en tanto que obra auténtica y útil a los demás lo que se impone, motor de un proyecto de mejor estar a nivel individual y colectivo lo que se recompone por la actividad por la actividad y frente a la pandemia, los vínculos entre los actores, reevalúa espontáneamente el valor de las cosas y de las ocupaciones. No hay, por supuesto, un proyecto societal explícito y primero en todo esto; sin duda es simplemente una inyección de lo real, irresistible, de hacer una obra común. Pero, ¿cómo no ver en lo que producen estas inteligencias en obra, las llaves de una renovación de los sistemas de producción de bienes y servicios? En la crisis sanitaria y su urgencia, las normas habituales del día a día desaparecen, autorizando a cada cual a reinventar aquello en lo que antes estaba encerrado. "La ampliación del campo de acciones es una de las características típica y fundamental del desarrollo humano. [ ... ] La competencia de los trabajadores está muy ligada a su capacidad de cambiar de registro según las circunstancias" nos decía Wisner ${ }^{22}$, ergónomo. Las "subjetividades pesadas" ${ }^{23}$ de antes se convierten en subjetividades saludables ahora, en tanto que la utilidad de las economías locales y solidarias inscritas en sus territorios y los lugares de vida, el medio, rebela las verdaderas condiciones de subsistencia de cada uno y de sus prójimos.

En la crisis la vida se reinventa, la salud se construye, con y en los riesgos. Y es así que Canguilhem, médico y filósofo, resistente de otra crisis mundial entre 1939 y 1945, definía la salud: "Yo me siento bien en la medida en la que me siento capaz de mantener la responsabilidad de mis actos, de llevar las cosas a su existencia y de crear entre las cosas relaciones que no sucederían sin mí, y que no serían lo que son sin ellas"24.

Estas experiencias de trabajo convocan, de hecho, lo que dicen desde hace mucho tiempo la ergonomía de la actividad y otras disciplinas que se interesan en el trabajo:

- La importancia de considerar la actividad de aquellas y aquellos que la hacen, en sus dimensiones indisociablemente singulares, colectivas y societales. Son raros los cuidadores que ejercen su derecho de retirarse hoy frente a la penuria de camas, de personal, de mascarillas, de blusas, de geles, etc. ¿Qué riesgo superior al de contraer el Covid19 pueden correr? En este consentimiento con la exposición, en un contexto en el que las obligaciones en materia de seguridad y de protección de los trabajadores permanecen pese a todo, encontramos el debate y la complementariedad de los enfoques higienistas y constructivo de la salud ${ }^{25}$;

- La importancia de cruzar los puntos de vista, de asociar a las personas concernidas para construir el problema en su complejidad y como eje de toda orientación de la investigación que busca respuestas pertinentes, porque se ajustan a la realidad;

- La importancia para los trabajadores de contar para algo en la utilización que el trabajo hace de ellos mismos ${ }^{26}$. de retomar el control.

\section{Cualquier ciudadano es responsable de concebir esta pandemia, como una avería o como una crisis, para y más allá del Trabajo.}

Se puede concebir lo que sucede como una avería o como una crisis. Una avería es un accidente en el camino. Lleva, después del diagnóstico, a reparar o reemplazar las piezas defectuosas, incluso a reforzar el mantenimiento preventivo... para asegurar, en el futuro, la continuidad del sistema y garantizar su resiliencia. Entonces, lo que ha organizado la historia más reciente de nuestra sociedad perdurará hasta la siguiente avería, que puede ser más grave aún, y que habrá que de nuevo escoger entre concebirla como una avería o como una crisis. Si concebimos esta pandemia como una crisis, se torna entonces en una oportunidad de refundar, de redefinir aquello que nos importa, lo que vale para concebir lo que prevalecerá en los arbitrajes que lleguen. ¿Cuáles serán las capacidades y con qué criterios los autores del trabajo serán evaluados? La ocasión es sin duda propicia para el enriquecimiento de los criterios sobre la base de los cuales los logros de las organizaciones serán evaluados, hacia una toma en consideración bienvenida de la complejidad de las bazas humanas del trabajo, y del estatuto de lo humano en la producción de esos logros.

Wisner, A. (1997): “Aspects pychologiques de l'anthropotechnologie”, Le Travail Humain, 1997, 60,3, 229-254, p.250-251.

G. Le Blanc (2004), Les maladies de l'homme normal, París, Vrin.

G. Canguilhem (2002), Écrits sur la médecine, París, Seuil, p. 68.

P. Falzon(dir.) (2013) Ergonomie constructive, Paris, Puf.

Schwartz, Y., Durrive, L. (2003): «L'usage de soi comme nécessaire arbitrage entre 'l'usage de soi par soi et l'usage de soi par les autres'», Travail et ergologie, Toulouse, Octarès. 
Avería o crisis, habrá esfuerzos y dificultades que alcanzarán desigualmente a unos y otros. Pero puesto que en este caos una rara oportunidad se dibuja, ¿qué deseamos nosotros? ¿Para qué trabajo y qué sociedad vamos a obrar, es decir crear obra, cuando llegue el desconfinamiento? ¿Estaremos con la capacidad de valorizar lo que nos lleva a ver esperanza en esta actualidad dramática, de obrar para una renormalización mayor de nuestros terrenos de Trabajo y de Vida, esos territorios en el cruce de las bazas de la alimentación, de los cuidados, del hábitat, de la energía, del entorno, de la educación, de la ciencia y de la cultura?

Los ergónomos, especialistas del trabajo, tienen y tendrán que formar parte de los análisis y las experimentaciones de mañana, más allá de los apoyos de solidaridad en los cuales participan hoy en el corazón de este periodo en el que el tiempo parece suspendido. Ellas y ellos pueden ser modestamente y respetando su deontología, actores preciosos de los cambios que vendrán.

Si esta pandemia es un hecho social total, los diferentes sujetos societales del momento deben pensarse como conjunto. Y ningún sujeto escapa verdaderamente ni al Trabajo, ni al Territorio: la igualdad de acceso a los servicios públicos, la penosidad y la edad de jubilación, la precarización de los empleos, en lugar y puesto de los oficios, la representación y el diálogo social, la prevención de riesgos, etc. La crisis del hospital público, sin respuesta satisfactoria a los ojos de los trabajadores y trabajadoras de la salud, cristalizaba ella sola un buen número de cuestiones. Estos sujetos ahogados por la urgencia sanitaria, volverán mañana, puede que más violentamente, sobre todo porque esta crisis sanitaria se convertirá en una crisis económica y luego de empleo. La cuestión no es solamente la de nuestro devenir, adultos de hoy, sino la del porvenir de las generaciones futuras. Por tanto: ¿qué trabajo para qué sociedad? Sobre el hilo de equilibrio pandémico, tenemos, de un lado la avería, y del otro la crisis... Y tenemos que elegir.

\section{Bibliografía}

Canguilhem, G. (2002), Écrits sur la médecine, París, Seuil, p. 68

Curie, J. , Hajjar, V., Baubios-Broye, A. (1990): «Psychopathologie du Travail ou dérégulation du système des activités» Perspectives psychiatriques, 22, pp. 85-91.

Daniellou, F. (2006): «Je me demanderais ce que la société attends de nous...», A propos des positions éptismologiques d’Alain Wisner, Travailler, 15,2006,23-28.

Falzon, P. (dir.) (2013) Ergonomie constructive, Paris, Puf.

Friedmann G. (1956), Le Travail en miettes, Gallimard, (réed. 1964).

Clot, Y: (2010), Le Travail à cœur, Paris, La Découverte.

Le Blanc, G. (2004), Les maladies de l’homme normal, París, Vrin.

Mauss,M. (1973): «Essai sur le don: forme et raison de l'échange dans les sociétés archaï ques», en Sociologie et Anthropologie, PUF, Collection Sociologie d'aujourd'hui, 1973, 143-279, pp. 274-275

Messing, K., Haentjens, C. y Doniol-Shaw, G.: "L'invisible nécessaire: l'activité de nettoyage des toilettes sur les trains de de voyageurs en gare", Le Travail Humain, 55, 4, 1992, pp. 353-370.

Schwartz, Y., Durrive, L. (2003) : «L'usage de soi comme nécessaire arbitrage entre 'l'usage de soi par soi et l'usage de soi par les autres'», Travail et ergologie, Toulouse, Octarès.

Teiger, C. (1993) : "L'approche ergonomique: du Travail humain à l'activité des hommes et des femmes au travail, Éducation Permanente, 1993, 116, pp.71-96

Wisner, A. (1997): “Aspects pychologiques de l'anthropotechnologie”, Le Travail Humain, 1997, 60,3, 229-254, p.250-251. 